\title{
Article
}

\section{Exploring Phenotypic and Genetic Overlap Between Cannabis Use and Schizotypy $\ddagger$}

\author{
James Vaissiere ${ }^{1, \star}$, Jackson G. Thorp ${ }^{1,2, \star}$, Jue-Sheng Ong $^{3}$, Alfredo Ortega-Alonso ${ }^{4,5}$ and Eske M. Derks ${ }^{1,6}$ \\ ${ }^{1}$ Translational Neurogenomics, QIMR Berghofer Medical Research Institute, Brisbane, Queensland, Australia, ${ }^{2}$ Faculty of Medicine, University of Queensland, \\ Brisbane, Queensland, Australia, ${ }^{3}$ Statistical Genetics, QIMR Berghofer Medical Research Institute, Brisbane, Queensland, Australia, ${ }^{4}$ Department of Psychology \\ and Logopedics, Faculty of Medicine, University of Helsinki, Helsinki, Finland, ${ }^{5}$ Department of Public Health Solutions, National Institute for Health and Welfare, \\ Helsinki, Finland and ${ }^{6}$ School of Biomedical Sciences, Faculty of Health, Queensland University of Technology, Brisbane, Queensland, Australia
}

\begin{abstract}
There is a well-established relationship between cannabis use and psychosis, although the exact nature of this relationship is not fully understood. Recent studies have observed significant genetic overlap between a diagnosis of schizophrenia and lifetime cannabis use. Expanding on this work, the current study aimed to examine whether genetic overlap also occurs for subclinical psychosis (schizotypy) and cannabis use, as well as examining the phenotypic association between the traits. Phenotypic correlations were calculated for a variety of schizotypy and cannabis phenotypes in the UK Biobank (UKB), and single nucleotide polymorphism (SNP)-based heritability estimates and genetic correlations were calculated for these UKB phenotypes as well as for several other variables taken from recent genomewide association studies. Positive phenotypic correlations were observed between 11 out of 12 pairs of the cannabis use and schizotypy phenotypes (correlation range .05-.18), indicating a robust association between increased symptoms of schizotypy and cannabis use. SNP-based heritability estimates for two schizotypy phenotypes remained significant after multiple testing correction: social anhedonia $\left(h_{\mathrm{SNP}}^{2}=.08, S E=.02, N=4025\right)$ and ever seen an unreal vision $\left(h_{\mathrm{SNP}}^{2}=.35, S E=.10, N=150,717\right)$. Finally, one significant genetic correlation was observed between schizotypy and cannabis use, a negative correlation between social anhedonia and number of times used cannabis $\left(r_{\mathrm{g}}=-.30, p=.012\right)$. The current study suggests the relationship between cannabis use and psychosis is also seen in subclinical symptoms of psychosis, but further research with larger samples is needed to determine the biological mechanisms underlying this association.
\end{abstract}

Keywords: Schizotypy; subclinical psychosis; symptoms; cannabis; genetic overlap

(Received 23 June 2020; accepted 23 July 2020)

Psychosis is a term used to describe symptoms characterized by the loss of contact with reality. Psychotic symptoms are a core feature of several common psychiatric disorders, most notably schizophrenia and bipolar disorder (van Os et al., 2009). Psychosis most often develops in late adolescence and has been considered to be typical of the late stages of these disorders (Insel, 2010). Psychotic disorders affect a significant number of individuals in the population and are one of the costliest groups of disorders in terms of medical expenditure (Fineberg et al., 2013; Wittchen \& Jacobi, 2005). Current evidence suggests that the risk of psychosis is influenced by both genetic and environmental factors (Dean \& Murray, 2005; Ortega-Alonso et al., 2017; Tienari et al., 2004).

In the past, psychosis has often been described by clinicians in dichotomous terms, where it is assumed to exist only at two levels: present and absent (Verdoux \& van Os, 2002). However, it is now

Author for correspondence: Jackson Thorp, Email: Jackson.Thorp@qimrberghofer.edu.au *These authors contributed equally to this work.

$\ddagger$ The original version of this article was submitted with an incorrect author name. A notice detailing this has been published and the error rectified in the online PDF and HTML copies.

Cite this article: Vaissiere J, Thorp JG, Ong J-S, Ortega-Alonso A, and Derks EM. (2020) Exploring Phenotypic and Genetic Overlap Between Cannabis Use and Schizotypy. Twin Research and Human Genetics 23: 221-227, https://doi.org/10.1017/ thg. 2020.68 established that psychosis can be better explained on a continuum scale of liability, with levels of the symptom ranging from relatively mild up to clinical diagnostic levels (Eaton et al., 1991; Grant et al., 2015; van Os et al., 1999, 2000, 2009; Verdoux \& van Os, 2002). In much of the current literature, the term schizotypy is used to describe the subclinical levels of psychotic symptoms. Some of the most common symptoms of schizotypy include physical and social anhedonia, delusions, hallucinations and hypomanic personality (Chapman et al., 1976, 1978; Eckblad \& Chapman, 1986). In an effort to maximize treatment outcomes, a significant body of research has attempted to explain the etiology of schizotypic symptomology. Genetic studies have offered a promising avenue for this, both in terms of twin and family studies as well as studies of molecular genetics. A large number of family and twin studies have suggested a substantial contribution of genetic factors for schizotypic symptomology. One early twin study including 29 twin pairs classified symptoms of schizotypy into four factors: positive and negative symptom schizotypy, positive trait schizotypy and trait anhedonia (Kendler et al., 1991). Moderate to high heritability estimates were found for three of the four factors, although findings should be interpreted cautiously due to sample size limitations. A follow-up twin study by Kendler and Hewitt (1992) involving more twin pairs ( $n=409$ pairs) showed that genetic

(C) The Author(s) 2020. Published by Cambridge University Press. This is an Open Access article, distributed under the terms of the Creative Commons Attribution licence (http:// creativecommons.org/licenses/by/4.0/), which permits unrestricted re-use, distribution, and reproduction in any medium, provided the original work is properly cited. 
Table 1. Overview of cannabis use and schizotypy phenotypes and SNP-based heritability estimates

\begin{tabular}{|c|c|c|c|c|}
\hline Construct & Phenotype & Source & $N$ & $\mathrm{~h}_{\text {SNP }}^{2}(\mathrm{SE})$ \\
\hline \multirow{10}{*}{ Psychosis/schizotypy } & Hypomanic personality & Ortega-Alonso et al. (2017) & 3967 & $.20(.12)$ \\
\hline & Perceptual aberration & Ortega-Alonso et al. (2017) & 4057 & $.09(.13)$ \\
\hline & Physical anhedonia & Ortega-Alonso et al. (2017) & 3988 & $.24(.12) *$ \\
\hline & Social anhedonia & Ortega-Alonso et al. (2017) & 4025 & $.35(.10)^{* *}$ \\
\hline & Distress caused by psychotic experience ${ }^{a}$ & UKB & 7290 & $.03(.06)$ \\
\hline & Frequency of psychotic experience (last year) ${ }^{a}$ & UKB & 7290 & $.05(.06)$ \\
\hline & Ever believed in an unreal conspiracy & UKB & 151,979 & $.06(.06)$ \\
\hline & Ever believed in unreal communication or signs & UKB & 151,909 & $.09(.06)$ \\
\hline & Ever seen an unreal vision & UKB & 150,717 & $.08(.02)^{* *}$ \\
\hline & Ever heard an unreal voice & UKB & 151,644 & $.05(.03)$ \\
\hline \multirow[t]{4}{*}{ Cannabis use } & Lifetime cannabis use & Pasman et al. (2018) & 162,082 & $.11(.01)^{* *}$ \\
\hline & Cannabis use disorder & Demontis et al. (2019) & 51,372 & $.13(.05) *$ \\
\hline & Number of times used cannabis ${ }^{a}$ & UKB & 152,145 & $.07(.01)^{* *}$ \\
\hline & Maximum frequency of cannabis use $\mathrm{a}^{\mathrm{a}}$ & UKB & 35,522 & $.05(.02) * *$ \\
\hline
\end{tabular}

Note: UKB, UK Biobank. ${ }^{\star} p<.05 ;{ }^{* \star} p<3.57 \times 10^{-3}$ (Bonferroni corrected threshold).

${ }^{a}$ Ordinal-scale phenotypes.

factors played a strong role in all psychosis symptoms except bodyimage perceptual aberration, with heritability estimates ranging between $29 \%$ and $68 \%$. A more recent study by Zavos et al. (2014), including 5059 twin pairs yielded similar findings, reporting heritability estimates between $15 \%$ and $59 \%$ for all symptoms except mild hallucinations, which was not significantly influenced by genetic factors. These studies therefore suggest that genetic influences play an important role for most schizotypy symptoms.

Several genomewide association studies (GWAS) have been performed to investigate the molecular genetic underpinnings of schizotypy (Legge et al., 2019; Ortega-Alonso et al., 2017; Tomppo et al., 2012). A study of 4269 nonpsychotic participants assessed genetic associations for four key symptoms of schizotypy: hypomanic personality, perceptual aberration, physical anhedonia and social anhedonia (Ortega-Alonso et al., 2017). One genomewide significant locus that was associated with hypomanic personality was identified, as well as 20 significant gene-based associations. Single nucleotide polymorphism (SNP)-based heritability estimates $\left(\mathrm{h}^{2}{ }_{\mathrm{SNP}}\right)$ were all significant and ranged between $16.6 \%$ and $27.4 \%$, demonstrating an influence of common SNPs on the measured symptoms of schizotypy. A recent GWAS in up to 127,966 participants from the UK Biobank (UKB) identified two genomewide significant genomic loci for any psychotic experience ( $n$ cases $=6123$ ) and two loci for distressing psychotic experience ( $n$ cases $=2143$; Legge et al., 2019). Overall, based on molecular genetic and family studies, the consensus held in the literature is that genetic differences exist among individuals that interact with environmental factors to determine whether an individual crosses the threshold for a clinical diagnosis of a psychotic disorder (Ortega-Alonso et al., 2017; Tienari et al., 2004). In the context of this, improved knowledge of the risk factors (genetic or environmental) that may increase an individual's liability to experience psychosis is imperative.

A number of risk factors over and above the genetic factors previously discussed appear to increase an individual's predisposition for schizotypy (McDonald \& Murray, 2000). One risk factor that has received considerable attention is cannabis use, a trait that has been established to have a partial genetic basis (Pasman et al., 2018; Stringer et al., 2016; Verweij et al., 2010). Earlier studies established an association between cannabis use and various levels of psychosis, including schizophrenia and schizotypy. These studies reported that patients with schizophrenia are more likely to use cannabis than other psychiatric patients and controls, and that using cannabis is associated with an increased likelihood of developing psychotic symptoms by a factor between 1.7 and 3.2 (Di Forti et al., 2019; Hall et al., 2004). On top of this, the rate of cannabis use disorder (CUD) is approximately $27 \%$ for patients with schizophrenia (Koskinen et al., 2009). This is in contrast to the rates in the general population, which is estimated to be around $1 \%$ (Copeland \& Swift 2009). Similarly, for schizotypy, positive correlations have been found between various measures and cannabis use, as well as for many of the aforementioned symptoms (Bailey \& Swallow 2004; Dumas et al., 2002; Mason et al., 2008; Richardson \& Garavan, 2011; Williams et al., 1996).

Molecular genetic studies have found evidence for a shared genetic architecture between cannabis use and schizophrenia (Pasman et al., 2018; Power et al., 2014; Verweij et al., 2017). Using Mendelian randomization analysis, Pasman et al. (2018) provided evidence for a causal influence of high genetic liability for schizophrenia on cannabis use. No evidence was found for a causal effect of cannabis use on schizophrenia, but this may be due to a lack of power. Expanding on these studies, the current study aims to further elucidate the relationship between psychosis and cannabis use by exploring genetic overlap between different aspects of schizotypy and cannabis use.

\section{Materials and Methods}

\section{Phenotypes}

In the current study, a total of 10 psychosis and four cannabis phenotypes were analyzed. Genomewide association analyses were conducted on six psychosis and two cannabis phenotypes within the UKB. The remaining phenotypes were operationalized using GWAS summary statistics from Demontis et al. (2019), OrtegaAlonso et al. (2017), and Pasman et al. (2018). An overview of all phenotypes analyzed is provided in Table 1 . For the UKB phenotypes, participants were included in the sample if they were of 
white British ancestry, identified through self-reported ethnicity and ancestral principal components (for more detail see MacGregor et al., 2018). Participants with a diagnosis of any psychotic illness were excluded to minimize confounding bias, as the current study was interested only in subclinical levels of psychosis. See Supplementary Tables 1 and 2 for a detailed description of each phenotype of interest and their respective response frequencies in the UKB.

\section{Schizotypy}

Within the UKB, six schizotypy phenotypes were analyzed: 'distress caused by psychotic experience', 'frequency of psychotic experience', 'ever seen an unreal vision', 'ever believed in unreal communication', 'ever heard an unreal voice' and 'ever believed in an unreal conspiracy against self. Each phenotype was measured using a single question administered to participants in the UKB online mental health questionnaire (Davis et al., 2020). Responses to each item were either binary, indicating the presence or absence of the behavior/symptom in question, or ordinal, indicating increasing levels of the symptom or behavior. The only exception to this was the item 'distress caused by psychoticexperience', for which responses fit a nominal scale. Initially, each response was coded $0-4$, with the 0 response representing not distressing, positive experience and 1 representing the response not distressing, neutral experience, while 2-4 represented increasing degrees of distress. The responses were recoded such that not distressing, positive experience and not distressing, neutral experience were combined into a single response, so that the phenotype was on an ordinal scale.

For the remaining four schizotypy phenotypes, we used GWAS summary statistics from Ortega-Alonso et al. (2017) of perceptual aberration, hypomanic personality, physical anhedonia and social anhedonia. Participants were Finnish citizens surveyed at 31 years of age who were part of the National Finnish Birth Cohort 1966. Participants were administered Chapman's schizotypia scales: the Revised Physical Anhedonia Scale, the Revised Social Anhedonia Scale, the Perceptual Aberration Scale and the Hypomanic Personality Scale (Chapman et al., 1976, 1978; Eckblad \& Chapman, 1986). Each of these scales utilizes a number of true/false items to assess the symptom in question, producing a cumulative score that measures the severity of that symptom. Both the reliability and validity of all of these scales have been well established in the literature (Bailey et al., 1993; Chan et al., 2015).

\section{Cannabis Use}

Two self-reported cannabis use phenotypes from the UKB were analyzed: 'number of times used cannabis' and 'maximum frequency of cannabis use', measured using a single question administered to participants in the UKB online mental health questionnaire. Both phenotypes were measured on an ordinal scale. Additionally, we used GWAS summary statistics of CUD by Demontis et al. (2019) with a sample size of 51,372 (2387 cases, 48,985 controls). Participants were all part of the iPSYCH database in Denmark, and cases were defined as having an International Classification of Diseases, Tenth Revision diagnosis of a CUD as confirmed by the Danish Psychiatric Central Research Register. Finally, we used GWAS summary statistics of lifetime cannabis use from Pasman et al. (2018), which included cohorts from the international cannabis consortium and the UKB (excluding the 23 andMe cohort). The phenotype was derived using a variety of self-report items that assessed whether the participant had ever used cannabis over the course of their lives. We note that this phenotype was derived using the same UKB item as 'number of times used cannabis' and therefore are not independent indicators of cannabis use.

\section{Statistical Analyses}

Phenotypic correlations. Polychoric correlations were calculated for all pairwise combinations of the cannabis and psychosis variables within the UKB using the psych $\mathrm{R}$ package. To account for multiple testing, a Bonferroni correction was applied (28 tests performed and therefore $p<1.79 \times 10^{-3}$ ).

Genomewide association analyses. GWA analyses for each of the UKB phenotypes were conducted using a linear mixed model approach implemented via the BOLT-LMM software (Loh et al., 2015) to adequately account for population structure and cryptic relatedness. While BOLT-LMM assumes that the trait of interest is quantitative, for binary phenotypes we applied the mathematical transformation outlined in Lloyd-Jones et al. (2018) to convert the resultant SNP-association estimates into log $(O R)$ estimates. Analyses were limited to autosomal SNPs with high imputation quality (INFO score $\geq 0.80$ ) and a minor allele frequency of $1 \%$ or higher. Sex, age at baseline, batch and 20 principal components were included as covariates in the model.

SNP-based heritability. SNP-based heritability $\left(\mathrm{h}_{\mathrm{SNP}}^{2}\right)$ was calculated for all phenotypes using LD score regression (LDSC; Bulik-Sullivan, Loh et al. 2015). Given that lifetime cannabis use, CUD, 'ever seen an unreal vision', 'ever heard an unreal voice', 'ever believed in an unreal conspiracy' and 'ever believed in unreal communication' were coded as binary phenotypes, and estimates were estimated on the liability scale. The population prevalence of the UKB phenotypes was estimated from the UKB sample (i.e. we assume population prevalence $=$ sample prevalence). While the ordinal scale of some phenotypes complicates the direct interpretation of $\mathrm{h}_{\text {SNP }}^{2}$ estimates, whether the estimate is significantly different from zero is not affected by the underlying scale. A Bonferroni correction was applied to account for multiple testing, and as 14 tests were completed, the corrected threshold was $p<3.57 \times 10^{-3}$.

Genetic correlations. Cross-trait LDSC (Bulik-Sullivan, Finucane et al., 2015) was used to calculate genetic correlations $\left(r_{g}\right)$ between all pair-wise combinations of cannabis and psychosis phenotypes that were found to have strong evidence of being heritable, $p\left(\mathrm{~h}_{\mathrm{SNP}}^{2}\right)<.05$. Multiple testing correction was similarly applied by adjusting $p$ values based on false discovery rate (FDR) across all tests.

\section{Results}

\section{Phenotypic Correlations}

Polychoric correlations $(\rho)$ were calculated between all UKB cannabis and schizotypy phenotypes. All except two correlations were significant after correction for multiple testing $\left(p<1.79 \times 10^{-3}\right.$; see Figure 1 and Supplementary Table 3 ). The majority of correlations (11 out of 12) between schizotypy and cannabis use were positive ( $\rho$ range $=.05-.18$ ), indicating that increased cannabis use is predominately associated with increased levels of schizotypy. The largest correlation was between 'ever believed in a conspiracy against self and 'number of times used cannabis'. There was a 


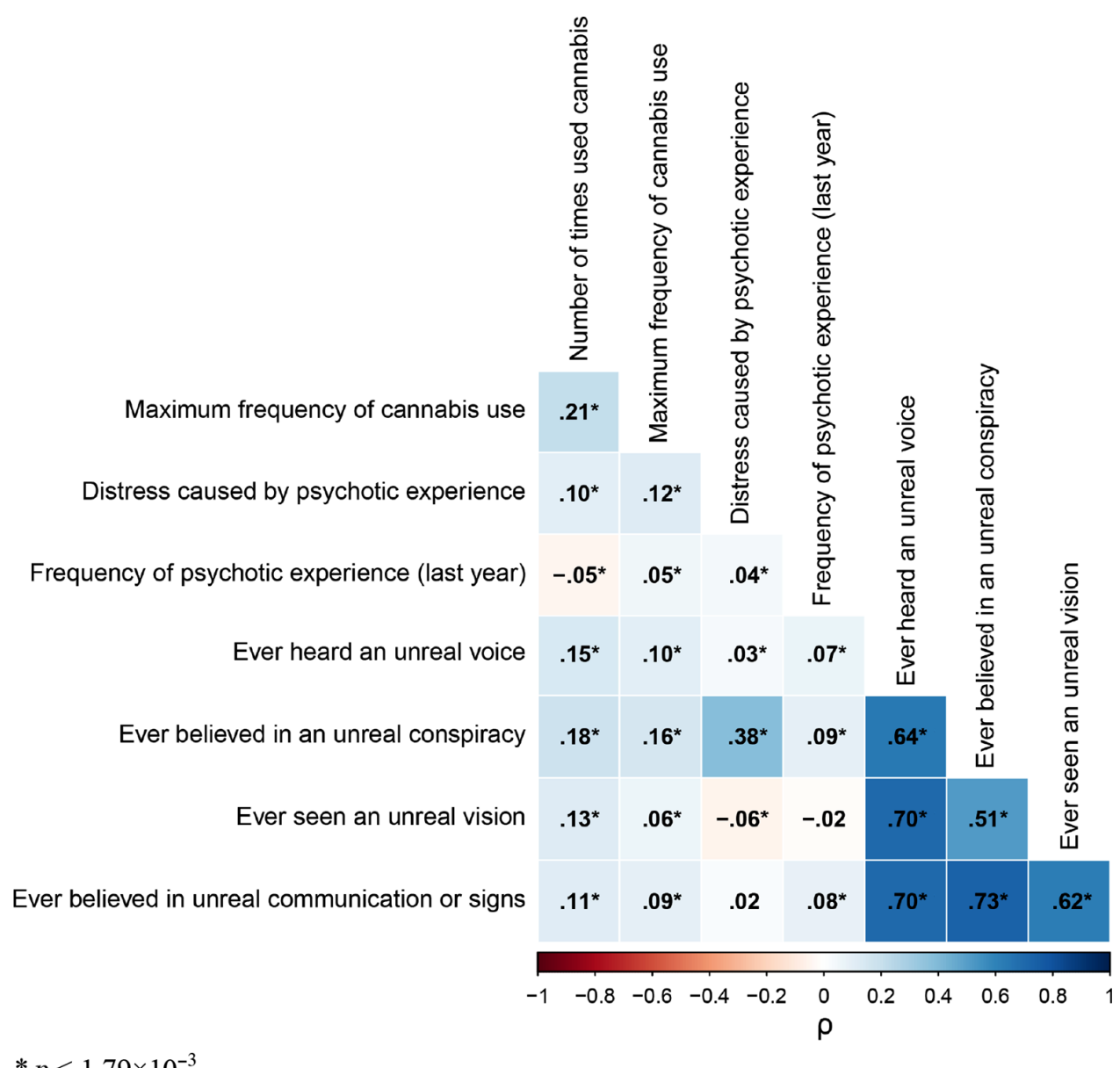

Fig. 1. Phenotypic correlations $(\rho)$ between

$* p<1.79 \times 10^{-3}$

small, negative correlation between 'frequency of psychotic experience' and 'number of times used cannabis' $(\rho=-.05)$.

\section{SNP-Based Heritability}

Using LDSC, individual SNP-based heritability estimates $\left(\mathrm{h}^{2}{ }_{\mathrm{SNP}}\right)$ were calculated for all phenotypes (see Table 1). All four cannabis phenotypes were found to have significant $h^{2}$ SNP estimates $(p<.05)$, ranging from .05 (maximum frequency of cannabis use) to .13 (cannabis use disorder). Three of these estimates (lifetime cannabis use, number of times used cannabis and maximum frequency of cannabis use) remained significant after correction for multiple testing $\left(p<3.57 \times 10^{-3}\right)$. Three psychosis phenotypes (physical anhedonia, social anhedonia and ever seen an unreal vision) had significant $\mathrm{h}_{\text {SNP }}^{2}$ estimates $(p<.05)$, ranging from .08 to .35 ; $\mathrm{h}^{2}{ }_{\text {SNP }}$ estimates for 'social anhedonia' and 'ever seen an unreal vision' remained significant after Bonferroni correction.

\section{Genetic Correlations}

Genetic correlations were calculated between all pairs of phenotypes that showed evidence of being heritable, resulting in genetic correlation analyses being performed between three psychosis phenotypes and four cannabis phenotypes (see Figure 2 and Supplementary Table 4). Genetic correlations between the cannabis phenotypes were all positive and significant $(p<.05)$, with $r_{\mathrm{g}} \mathrm{s}$ ranging from .33 to .87 . All but one of these correlations (cannabis use disorder and maximum frequency of cannabis use) remained significant after FDR correction. Within the psychosis phenotypes, only physical and social anhedonia were significantly genetically correlated $\left(r_{\mathrm{g}}=.66, p=.003\right)$. Between the cannabis use and psychosis phenotypes only one genetic correlation was significant: a negative correlation between social anhedonia and number of times used cannabis $\left(r_{\mathrm{g}}=-.30, p=.012\right.$. All remaining genetic correlations were not significant.

\section{Discussion}

The aim of the current study was to further unravel the relationship between psychosis and cannabis use by examining the phenotypic and genetic associations between subclinical levels of psychosis (i.e. schizotypy) and cannabis use. First, for all individual phenotypes, the amount of variance explained by common SNPs (SNP-based heritability) was estimated. Second, phenotypic correlations between various psychosis and cannabis phenotypes in the UKB cohort were calculated. Lastly, the proportion of genetic overlap between and within all cannabis and psychosis phenotypes was observed through genetic correlations.

Significant positive phenotypic correlations were observed between all cannabis use and schizotypy phenotypes. This demonstrates that for several specific symptoms of psychosis as well as for the distress caused by and the frequency of psychotic experience, there is a positive association with cannabis use and frequency of use. This finding is in line with the previous body of literature that has shown a positive association between cannabis use and schizotypy (Bailey \& Swallow, 2004; Dumas et al., 2002; Williams et al., 1996). Also of note is the finding that the cannabis use phenotypes 


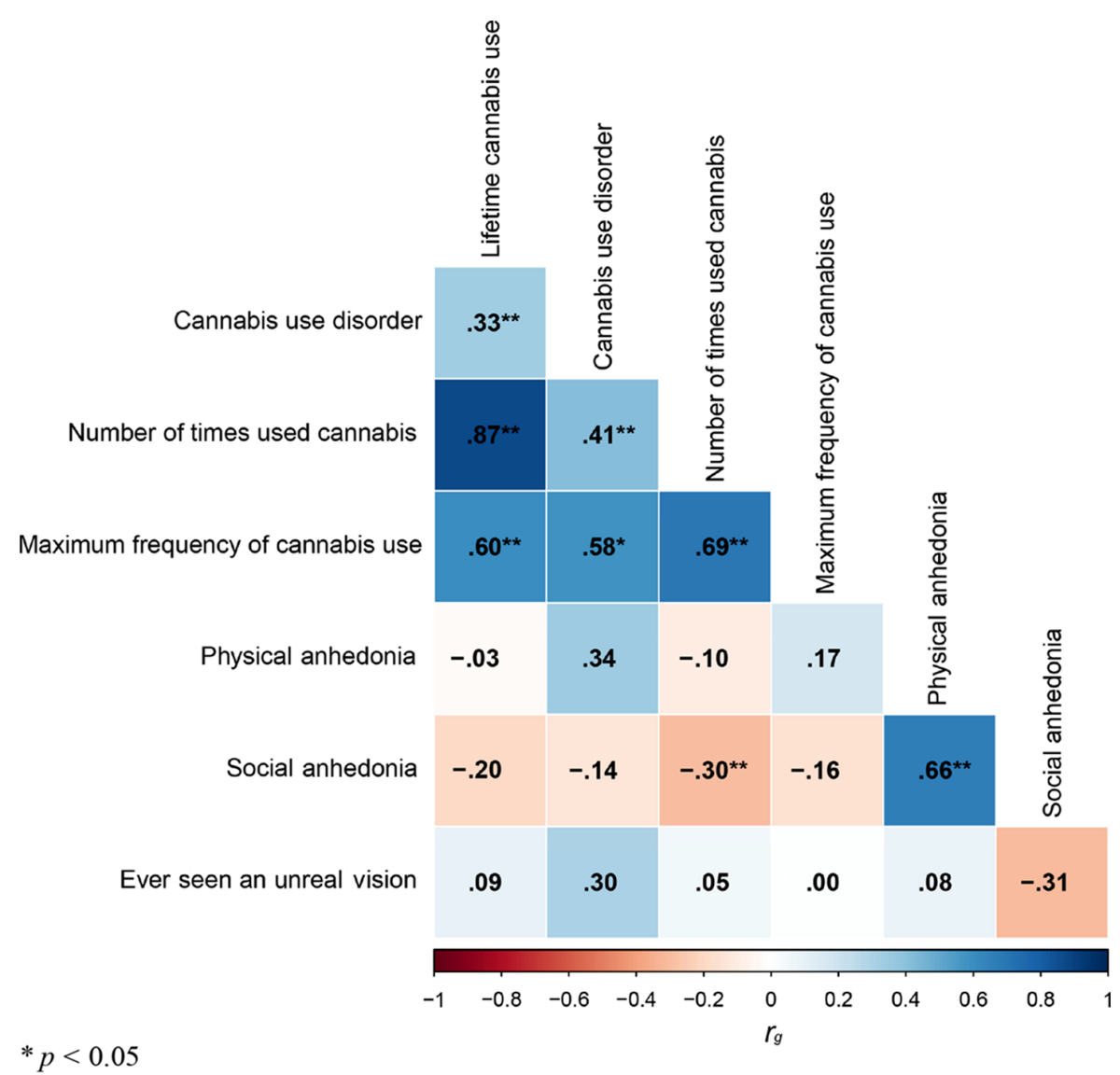

$* p<0.05$

Fig. 2. Genetic correlations $\left(r_{g}\right)$ between cannabis use

** FDR $<0.05$ and schizotypy phenotypes.

had the lowest correlations with frequency of psychotic experience, suggesting that cannabis use has a stronger link with ever having experienced psychosis than how often psychotic experiences occur. This is a novel finding, as the frequency of psychotic experience has not been measured against any dimension of cannabis use in the previous literature.

Second, while significant heritability estimates were observed for all cannabis phenotypes, the only psychosis phenotypes that were significantly heritable were physical anhedonia, social anhedonia and 'ever seen an unreal vision'. Finding significant heritability estimates for all cannabis phenotypes is in line with the previous literature (Pasman et al., 2018; Stringer et al., 2016; Verweij et al., 2010). The current SNP-based heritability estimates for the majority of the psychosis phenotypes were mixed in relation to the previous literature. Common SNPs did not appear to explain a significant amount of the variance in two of the phenotypes assessing hallucinations ('ever heard an unreal voice' or 'believed in unreal communication') in line with Zavos et al. (2014); they did, however, for 'ever seen an unreal vision'. Variance in both social and physical anhedonia also appeared to be significantly explained by common SNPs, which is what was found previously by Ortega-Alonso et al. (2017). In contrast to their findings, however, both hypomanic personality and perceptual aberration did not return significant heritability estimates. This is likely due to the fact that different methods were used to calculate SNP- $\mathrm{h}^{2}$; OrtegaAlonso et al. (2017) used genomewide restricted maximum likelihood, while the current study used LDSC due to only summary statistics being available. Finally, the remaining schizotypy phenotypes were not significantly influenced by common SNPs, in contrast to the previous literature suggesting the impact of genetic factors on psychotic symptoms. There are a number of possible explanations for the lack of a significant contribution of common SNPs. Common genetic factors may influence individual schizotypic symptoms to different degrees and thus those schizotypy measures that returned nonsignificant estimates in the current study are indeed not impacted to a large degree by common genetic variants. Another explanation is that the genetic contributions found in previous twin-based studies cannot be wholly explained by additive genetic effects or are instead due to rare SNPs. However, the most likely reason is that the current study was underpowered due to the relatively small sample sizes for schizotypic symptoms.

Among the trait pairs tested in our genetic correlation analyses, we only found evidence to support a common genetic basis between social anhedonia and number of times cannabis used $\left(r_{\mathrm{g}}=-.30\right)$. This suggests that common genetic risk factors that increase levels of social anhedonia are associated with lower levels of cannabis use. A possible explanation for this finding could be that within the current sample, smoking cannabis is mainly done within social settings and hence people who do not take pleasure from social settings do not engage in it as frequently. For the nonsignificant genetic correlation results, it could be that the genetic influences for the phenotypes assessed are genuinely separate, and thus no overlap exists between the genetic factors underpinning each phenotype. This would be in direct contrast to the findings of Pasman et al. (2018), who found a significant genetic correlation between a diagnosis of schizophrenia and lifetime cannabis use. A more plausible explanation is that again the current 
study was underpowered, as indicated by the fact that while many of the nonsignificant genetic correlations had large $r_{g}$ values, they also had relatively large standard errors. It should also be noted that Pasman et al. (2018) assessed a schizophrenia diagnosis, whereas the present study assessed individual symptoms. This difference in the level of analysis could be a reason for the contrasting findings, as the genes underpinning individual symptoms are likely not identical to the genes implicated in a broader diagnosis of an overarching disorder.

Lastly, significant positive genetic correlations were observed within all cannabis phenotypes and one pair of psychosis phenotypes (physical and social anhedonia). It therefore appears that there is a large overlap between the genetic factors that influence various aspects of cannabis use. Of note are the genetic correlations with CUD, while significant positive correlations were observed with all other cannabis phenotypes, the strongest correlation was with maximum frequency of use. This could have been observed for the simple reason that those who use cannabis regularly are at higher risk of developing CUD, as their increased use may be more prone to become problematic. With regard to the weak correlations within psychosis phenotypes, our findings reaffirm the notion that different psychotic symptoms may have different biological underpinnings. Physical and social anhedonia are likely to share the same biological causes, as they represent variations of the same symptom, namely reduced enjoyment in activities that previously brought pleasure (Chapman et al., 1976). The third phenotype, 'ever seen an unreal vision', represents an entirely different psychotic symptom, instead assessing a specific subset of hallucinations. As these symptoms differ considerably in their presentation, it is possible that each has a separate, distinct biological mechanism underlying it. Finally, it could be that again power was insufficient to detect a significant genetic correlation between these phenotypes.

\section{Limitations}

It is important to recognize the limitations of the current study. As previously mentioned, the primary limitation was inadequate power for some of the phenotypes. As multiple different samples were used and sample sizes varied, it is possible that the sample sizes for several phenotypes, such as those taken from OrtegaAlonso et al. (2017), were too small (Hong \& Park, 2012). Second, while all participants with a clinical diagnosis of a psychotic illness were excluded from the majority of samples, no other exclusion criteria were implemented so as not to reduce power further. Many of the symptoms assessed, such as anhedonia and hallucinations, can also be caused by conditions unrelated to psychosis (Ali et al., 2011; Anisman \& Matheson, 2005). It is therefore possible that the participants experiencing certain phenotypes in each sample were doing so as a result of a secondary diagnosis, and thus our GWA analyses might be tagging SNP associations implicated in these disorders and not exclusively on subclinical psychosis. Third, recruitment selection biases affecting the UKB cohort may have influenced our results (Munafò et al., 2017) and therefore replication in independent cohorts is necessary. Fourth, the number of times used cannabis phenotype included both users and nonusers, and thus we assume that the same genes underlie cannabis initiation and increasing levels of use. Finally, the measurements of the various aspects of schizotypy in the current study were relatively limited and difficult to quantify. To fully understand the relationship between psychosis and cannabis use, all symptoms of psychosis should be considered, which remains clinically challenging. For example, only one phenotype was measured that assessed delusions, and this assessed only one type of delusional belief. By not assessing all symptoms of schizotypy in the current analyses, the generalisability of the findings to psychosis is broadly limited.

\section{Implications and Future Research}

The variation in heritability estimates for psychosis phenotypes reinforces the notion that different aspects of schizotypy may have different biological mechanisms. It is important to examine schizotypy at the symptom level, as assessing psychosis as a single phenotype or trait may lead to inferences drawn that may not be generalizable across all symptoms. This symptom-level variation may be linked to clinical heterogeneity in psychosis, and individuals with differing symptomatology may be expected to respond to treatments differently. Future research should seek to replicate the current findings using larger studies with robust clinical endpoints. Additionally, any further research on the genetic relationship between cannabis and psychosis ought to include a wider range of psychotic symptoms to recognise the broad heterogeneity within psychotic symptomology and allow for more accurate inferences to be drawn.

Supplementary material. To view supplementary material for this article, please visit https://doi.org/10.1017/thg.2020.68.

Acknowledgments. We thank the research participants of all cohorts for making this study possible. This work was conducted using the UKB Resource (application number 25331). We would like to thank Stuart MacGregor and William Hennah for helpful discussions and feedback. We also thank William Hennah for sharing summary data of the National Finnish Birth Cohort 1966 (NFBC 1966). We also wish to acknowledge the work of the NFBC project center. This study included summary statistics of a genetic study on cannabis use (Pasman et al., 2018, Nature Neuroscience). We would like to acknowledge all participating groups of the International Cannabis Consortium, and in particular the members of the working group including Joelle Pasman, Karin Verweij, Nathan Gillespie, Eske Derks and Jacqueline Vink. Pasman et al. (2018) included data from the UK Biobank resource under application numbers 9905, 16406 and 25331.

Financial support. JGT is supported by a Research Training Scholarship from the University of Queensland. NFBC1966 received financial support from University of Oulu Grant no. 65354, Oulu University Hospital Grant no. 2/97, 8/97, Ministry of Health and Social Affairs Grant no. 23/251/97, 160/ 97, 190/97, National Institute for Health and Welfare, Helsinki Grant no. 54121 and Regional Institute of Occupational Health, Oulu, Finland Grant no. 50621,54231

\section{References}

Ali, S., Patel, M., Avenido, J., Jabeen, S., \& Riley, W. J. (2011). Hallucinations: Common features and causes. Current Psychiatry, 10, 22-29.

Anisman, H., \& Matheson, K. (2005). Stress, depression, and anhedonia: Caveats concerning animal models. Neuroscience \& Biobehavioral Reviews, 29, 525-546.

Bailey, B., West, K. Y., Widiger, T. A., \& Freiman, K. (1993). The convergent and discriminant validity of the Chapman scales. Journal of Personality Assessment, 61, 121-135.

Bailey, E. L., \& Swallow, B. L. (2004). The relationship between cannabis use and schizotypal symptoms. European Psychiatry, 19, 113-114.

Bulik-Sullivan, B., Finucane, H. K., Anttila, V., Gusev, A., Day, F. R., Loh, P.-R., ... Genetic Consortium for Anorexia Nervosa of the Wellcome Trust Case Control. (2015). An atlas of genetic correlations across human diseases and traits. Nature Genetics, 47, 1236-1241.

Bulik-Sullivan, B. K., Loh, P.-R., Finucane, H. K., Ripke, S., Yang, J., Patterson, N., ... Schizophrenia Working Group of the Psychiatric Genomics Consortium. (2015). LD score regression distinguishes 
confounding from polygenicity in genome-wide association studies. Nature Genetics, 47, 291-295.

Chan, R. C., Shi, H.-S., Geng, F.-l., Liu, W.-h., Yan, C., Wang, Y., \& Gooding, D. C. (2015). The Chapman psychosis-proneness scales: Consistency across culture and time. Psychiatry Research, 228, 143-149.

Chapman, L. J., Chapman, J. P., \& Raulin, M. L. (1976). Scales for physical and social anhedonia. Journal of Abnormal Psychology, 85, 374.

Chapman, L. J., Chapman, J. P., \& Raulin, M. L. (1978). Body-image aberration in Schizophrenia. Journal of Abnormal Psychology, 87, 399.

Copeland, J., \& Swift, W. (2009). Cannabis use disorder: Epidemiology and management. International Review of Psychiatry, 21, 96-103.

Davis, K. A. S., Coleman, J. R. I., Adams, M., Allen, N., Breen, G., Cullen, B., \& Hotopf, M. (2020). Mental health in UK Biobank $3 / 4$ Development, implementation and results from an online questionnaire completed by 157366 participants: A reanalysis. BJPsych Open, 6, e18.

Dean, K., \& Murray, R. M. (2005). Environmental risk factors for psychosis. Dialogues in Clinical Neuroscience, 7, 69.

Demontis, D., Rajagopal, V. M., Thorgeirsson, T. E., Als, T. D., Grove, J., Leppälä, K., \& Reginsson, G. W. (2019). Genome-wide association study implicates CHRNA2 in cannabis use disorder. Nature Neuroscience, 22, 1066-1074.

Di Forti, M., Quattrone, D., Freeman, T. P., Tripoli, G., Gayer-Anderson, C., Quigley, H., \& La Cascia, C. (2019). The contribution of cannabis use to variation in the incidence of psychotic disorder across Europe (EU-GEI): A multicentre case-control study. The Lancet Psychiatry, 6, 427-436.

Dumas, P., Saoud, M., Bouafia, S., Gutknecht, C., Ecochard, R., Daléry, J., \& d'Amato, T. (2002). Cannabis use correlates with schizotypal personality traits in healthy students. Psychiatry Research, 109, 27-35.

Eaton, W. W., Romanoski, A., Anthony, J. C., \& Nestadt, G. (1991). Screening for psychosis in the general population with a self-report interview. Journal of Nervous and Mental Disease, 179, 689-693.

Eckblad, M., \& Chapman, L. J. (1986). Development and validation of a scale for hypomanic personality. Journal of Abnormal Psychology, 95, 214-222.

Fineberg, N. A., Haddad, P. M., Carpenter, L., Gannon, B., Sharpe, R., Young, A. H., \& Nutt, D. J. (2013). The size, burden and cost of disorders of the brain in the UK. Journal of Psychopharmacology, 27, 761-770.

Grant, P., Munk, A. J. L., Kuepper, Y., Wielpuetz, C., \& Hennig, J. (2015). Additive genetic effects for schizotypy support a fully-dimensional model of psychosis-proneness. Journal of Individual Differences, 36, 87-92.

Hall, W., Degenhardt, L., \& Teesson, M. (2004). Cannabis use and psychotic disorders: An update. Drug and Alcohol Review, 23, 433-443.

Hong, E. P., \& Park, J. W. (2012). Sample size and statistical power calculation in genetic association studies. Genomics \& Informatics, 10, 117-122.

Insel, T. R. (2010). Rethinking schizophrenia. Nature, 468, 187-193.

Kendler, K. S., \& Hewitt, J. (1992). The structure of self-report schizotypy in twins. Journal of Personality Disorders, 6, 1-17.

Kendler, K. S., Ochs, A. L., Gorman, A. M., Hewitt, J. K., Ross, D. E., \& Mirsky, A. F. (1991). The structure of schizotypy: A pilot multitrait twin study. Psychiatry Research, 36, 19-36.

Koskinen, J., Löhönen, J., Koponen, H., Isohanni, M., \& Miettunen, J. (2009). Rate of cannabis use disorders in clinical samples of patients with schizophrenia: A meta-analysis. Schizophrenia Bulletin, 36, 1115-1130.

Legge, S. E., Jones, H. J., Kendall, K. M., Pardiñas, A. F., Menzies, G., Bracher-Smith, M., \& Walters, J. T. R. (2019). Association of genetic liability to psychotic experiences with neuropsychotic disorders and traits. JAMA Psychiatry, 76, 1256-1265.

Lloyd-Jones, L. R., Robinson, M. R., Yang, J., \& Visscher, P. M. (2018). Transformation of summary statistics from linear mixed model association on all-or-none traits to odds ratio. Genetics, 208, 1397-1408.

Loh, P.-R., Tucker, G., Bulik-Sullivan, B. K., Vilhjálmsson, B. J., Finucane, H. K., Salem, R. M., \& Price, A. L. (2015). Efficient Bayesian mixed-model analysis increases association power in large cohorts. Nature Genetics, 47 , 284-290.

MacGregor, S., Ong, J. S., An, J., Han, X., Zhou, T., Siggs, O. M., \& Hewitt, A. W. (2018). Genome-wide association study of intraocular pressure uncovers new pathways to glaucoma. Nature Genetics, 50, 1067-1071.
Mason, O. J., Morgan, C. J., Stefanovic, A., \& Curran, H. V. (2008). The psychotomimetic states inventory (PSI): Measuring psychotic-type experiences from ketamine and cannabis. Schizophrenia Research, 103, 138-142.

McDonald, C., \& Murray, R. M. (2000). Early and late environmental risk factors for schizophrenia. Brain Research Reviews, 31, 130-137.

Munafò, M. R., Tilling, K., Taylor, A. E., Evans, D. M., \& Davey Smith, G. (2017). Collider scope: When selection bias can substantially influence observed associations. International Journal of Epidemiology, 47, 226-235.

Ortega-Alonso, A., Ekelund, J., Sarin, A. P., Miettunen, J., Veijola, J., Jarvelin, M. R., \& Hennah, W. (2017). Genome-wide association study of psychosis proneness in the finnish population. Schizophrenia Bulletin, 43, 1304-1314.

Pasman, J. A., Verweij, K. J. H., Gerring, Z., Stringer, S., Sanchez-Roige, S., Treur, J. L., \& Vink, J. M. (2018). GWAS of lifetime cannabis use reveals new risk loci, genetic overlap with psychiatric traits, and a causal influence of schizophrenia. Nature Neuroscience, 21, 1161-1170.

Power, R. A., Verweij, K. J., Zuhair, M., Montgomery, G. W., Henders, A. K., Heath, A. C., \& Martin, N. G. (2014). Genetic predisposition to schizophrenia associated with increased use of cannabis. Molecular Psychiatry, 19, 1201-1204.

Richardson, T., \& Garavan, H. (2011). Relationships between substance use and hypomanic symptoms in a non-clinical sample. Mental Health and Substance Use, 4, 211-221.

Stringer, S., Minică, C., Verweij, K. J., Mbarek, H., Bernard, M., Derringer, J., Maciejewski, D. F. (2016). Genome-wide association study of lifetime cannabis use based on a large meta-analytic sample of 32330 subjects from the International Cannabis Consortium. Translational Psychiatry, 6, e769.

Tienari, P., Wynne, L. C., Sorri, A., Lahti, I., Läksy, K., Moring, J., \& Wahlberg, K.-E. (2004). Genotype-environment interaction in schizophrenia-spectrum disorder: Long-term follow-up study of Finnish adoptees. The British Journal of Psychiatry, 184, 216-222.

Tomppo, L., Ekelund, J., Lichtermann, D., Veijola, J., Järvelin, M.-R., \& Hennah, W. (2012). DISC1 conditioned GWAS for psychosis proneness in a large Finnish birth cohort. PLoS One, 7, e30643.

van Os, J., Hanssen, M., Bijl, R. V., \& Ravelli, A. (2000). Strauss (1969) revisited: A psychosis continuum in the general population? Schizophrenia Research, 45, 11-20.

van Os, J., Linscott, R. J., Myin-Germeys, I., Delespaul, P., \& Krabbendam, L. (2009). A systematic review and meta-analysis of the psychosis continuum: Evidence for a psychosis proneness-persistence-impairment model of psychotic disorder. Psychological Medicine, 39, 179-195.

van Os, J., Verdoux, H., Maurice-Tison, S., Gay, B., Liraud, F., Salamon, R., \& Bourgeois, M. (1999). Self-reported psychosis-like symptoms and the continuum of psychosis. Social Psychiatry and Psychiatric Epidemiology, $34,459-463$.

Verdoux, H., \& van Os, J. (2002). Psychotic symptoms in non-clinical populations and the continuum of psychosis. Schizophrenia Research, 54, 59-65.

Verweij, K. J., Zietsch, B. P., Lynskey, M. T., Medland, S. E., Neale, M. C., Martin, N. G., \& Vink, J. M. (2010). Genetic and environmental influences on cannabis use initiation and problematic use: A meta-analysis of twin studies. Addiction, 105, 417-430.

Verweij, K. J. H., Abdellaoui, A., Nivard, M. G., Sainz Cort, A., Ligthart, L., Draisma, H. H. M., \& Vink, J. M. (2017). Short communication: Genetic association between schizophrenia and cannabis use. Drug and Alcohol Dependence, 171, 117-121.

Williams, J., Wellman, N., \& Rawlins, J. (1996). Cannabis use correlates with schizotypy in healthy people. Addiction, 91, 869-877.

Wittchen, H.-U., \& Jacobi, F. (2005). Size and burden of mental disorders in Europe - A critical review and appraisal of 27 studies. European Neuropsychopharmacology, 15, 357-376.

Zavos, H. M., Freeman, D., Haworth, C. M., McGuire, P., Plomin, R., Cardno, A. G., \& Ronald, A. (2014). Consistent etiology of severe, frequent psychotic experiences and milder, less frequent manifestations: A twin study of specific psychotic experiences in adolescence. JAMA Psychiatry, $71,1049-1057$. 